\title{
Incidence d'une crue type 1910 sur l'exploitation de la SNCF
}

\author{
The effects of a 1910 type flood on SNCF working
}

par Pierre Pouillon

Direction Ile de France de la SNCF

Although floods do not happen with the same shape, considering a flood like that of 1910 illustrates strengths and weaknesses of the SNCF railway network in Ile de France. The most important part of the railways can work, but the trafic must be interrupted on different places situated on principal axes. The return to a normal working would not be very quick.

\section{CARACTÉRISTIQUES GÉNÉRALES DU PROBLÈME}

Les voies ferrées des grands réseaux qui ont constitué la SNCF ont été en général construites très au-dessus du niveau des crues, y compris une crue centennale du type de celle de 1910. La sensibilité aux inondations est donc à considérer sur une logique de points singuliers, chaque crue pouvant intéresser des points singuliers bien différents. Il sera très intéressant de rappeler, à titre d'exemple, les principaux points touchés en 1910, les conséquences sur l'exploitation pendant la crue, et enfin les conditions de remise en état du réseau. Enfin, nous examinerons de façon plus détaillée un cas particulier particulièrement sensible, non représentatif de l'ensemble du réseau en général peu inquiété, celui de la ligne $\mathrm{C}$ du RER dans Paris.

\section{II $\square$ LA CIRCULATION DES TRAINS LORS D'UNE CRUE CENTENNALE}

Les gares tête de ligne de Paris restent hors d'eaux à l'exception de la gare d'Austerlitz. Les conditions de circulation aux environs de Paris sont les suivantes :

- Les voies sont sous l'eau de Chelles à Meaux sur l'axe Paris-Strasbourg par suite du refoulement des eaux de la Seine dans la Marne. La circulation est normale sur la relation Paris-Gretz-Troyes-Bâle.

- Les grandes relations au départ de la gare du Nord sont normalement desservies, sauf les relations Paris-Dieppe, qui ont à souffrir des débordements de l'Oise, par refoulement des eaux de la Seine, près de Pontoise.

- Les abords de la gare Saint-Lazare ont été en 1910 inondés par des eaux amenées par les réseaux souterrains. Les progrès qu'a pu connaître la protection des réseaux souterrains sera un élément déterminant pour maintenir libre l'accès des piétons à une gare Saint-Lazare qui de toute façon reste hors d'eaux. Entre Paris et Mantes, les voies sont recouvertes de quelques dizaines de centimètres d'eau en certains points, en gare du Stade, de Poissy à Vernouillet-Verneuil, en gare des Mureaux et d'Epône à Mézières.

- La gare Montparnasse et les voies qui en partent à destination de l'ouest et du sud-ouest ne sont pas concernées et restent exploitables normalement.

- Les voies de la gare d'Austerlitz sont submergées, à Austerlitz même, ainsi que par endroit jusqu'à Juvisy, avec des hauteurs d'eau variant de trente à cent cinquante centimètres.

- Les voies du sud-est sont inondées de Maisons-Alfort à Villeneuve, puis entre Villeneuve et Corbeil avec des hauteurs d'eau dépassant un mètre cinquante.

- Le réseau à grande vitesse est normalement accessible depuis les gares du Nord et Montparnasse.

\section{III $\square$ LES CIRCULATIONS APRÈS LA CRUE}

Une remise en circulation rapide, soit quelques jours, est envisageable sur les axes qui ont été concernés, ce délai correspondant au rétablissement des désordres qui auront pu être causés à l'infrastructure. Soulignons la différence fondamentale entre le bassin de la Seine en amont et en aval de Paris.

En aval, les hauteurs d'eau ne seraient que de quelques dizaines de centimètres. On peut espérer que les mesures préventives prises ces dernières décennies seraient à même d'éviter toute inondation importante. En tout état de cause, 
les dégâts aux installations seraient de faible ampleur et un rythme quasi-normal des circulations est envisageable dès le rétablissement de la continuité des itinéraires.

En amont de Paris, le long de la Seine et de la Marne, les hauteurs d'eau ont atteint en 1910 en certains points $1,50 \mathrm{~m}$ au dessus des rails. Les zones inondées sont beaucoup plus importantes, les installations de signalisation, d'alimentation en énergie seraient en de nombreux points devenues inutilisables. La réparation des désordres de l'infrastructure serait là aussi de quelques jours, permettant ainsi un rétablissement des circulations en nombre réduit. La remise en état complète du réseau ne serait par contre que très progressive, et étroitement dépendante des possibilités et des délais de livraison des industries qui fournissent les installations de signalisation, de transmission et d'énergie électrique. Remarquons cependant là aussi que tout gain sur la hauteur de crue, même s'il n'empêche pas l'interruption des circulations ferroviaires, accélère la remise en état complète en limitant l'étendue des installations électriques à remplacer. Citons un exemple significatif : un gain d'un mètre d'eau permet d'éviter l'inondation de la rue de Bercy et donc de la gare souterraine de Paris-Lyon, de la partie centrale des RER D et A à partir de la salle Méditerranée qui relie la rue de Bercy à la rue de Chalon.

\section{IV — UN CAS PARTICULIER : D'AUSTERLITZ A JUVISY ET LA LIGNE C DANS PARIS}

Contrairement au reste du réseau aux environs de Paris, cette section de ligne est plus particulièrement exposée aux risques de crue, et à des niveaux inférieurs à la crue centennale.

Rappelons que les voies menant à la gare d'Orsay, qui ont été intégrées dans le RER C, ont été noyées en 1910, 1920, $1924,1945,1955$, alors même que le reste du réseau était peu ou pas inquiété, pour des hauteurs de crue allant de 6,10 à $6,50 \mathrm{~m}$ au pont d'Austerlitz. Les mesures à prendre en cas de crue font donc l'objet de consignes très détaillées et régulièrement tenues à jour. Les leviers d'action essentiels sont : - la vérification périodique du fonctionnement d'installations de pompage et de l'approvisionnement de stocks de matériaux destinés à la construction de batardeaux,

- la surveillance du niveau de l'eau de la Seine et la connaissance des évolutions prévisibles et décisions corrélatives d'arrêter l'exploitation et de déposer des installations sensibles comme les installations de signalisation ou d'alimentation électrique,
- la liaison avec la ville de Paris et la RATP pour assurer une bonne coordination des mesures et une application sans faille,

- la mise en place des batardeaux aux endroits prévus pour découper la ligne en différents biefs,

- en gare d'Orsay, charger les voies par des trains de cailloux pour garantir la stabilité du radier jusqu'à un certain niveau de crue,

- assurer la stabilité des ouvrages par une inondation sélective et maîtrisée.

Sans être exhaustif, citons quelques cotes significatives au pont d'Austerlitz. Dès une hauteur de $1,80 \mathrm{~m}$, une surveillance spéciale des installations est déclenchée. A $3,00 \mathrm{~m}$, les portes et vannes entre nos installations et la Seine commencent à être progressivement fermées. A $5,00 \mathrm{~m}$, les trains de matériaux destinés au lestage du radier sont mis en place à proximité immédiate. Une décision difficile à prendre est l'arrêt du fonctionnement de la ligne $\mathrm{C}$ d'Austerlitz à Boulevard Victor. Cette décision est prise pour une cote de l'ordre de $6,00 \mathrm{~m}$ au pont d'Austerlitz et en fonction de l'évolution prévisible de la montée des eaux. Le lestage du radier et la construction de batardeaux sont aussitôt entrepris. L'inondation sélective et maîtrisée commence à une cote de $6,90 \mathrm{~m}$ après dépose d'un maximum d'installations ferroviaires (signalisation, télécommunications, énergie...).

Les voies de surface de la gare de Paris Austerlitz ne sont plus exploitées à partir d'une hauteur d'eau de 7,68 m, cote à laquelle un barrage doit être mis en place à Masséna pour protéger la ville en fonction, là aussi, des prévisions d'évolution de la crue. La gare de Paris Austerlitz ne court cependant un risque d'inondation que pour une crue de plus de huit mètres. C'est à partir de ce seuil que les installations ferroviaires sont envahies par les eaux en amont de Paris, à Vitry, à Choisy et à Juvisy. En effet, à la suite de l'inondation de 1910, des ouvrages de protection ont été construits pour assurer l'exploitation le plus longtemps possible en cohérence avec le seuil de 7,68 m fixé pour l'exploitation de la gare d'Austerlitz.

Nous voyons donc que cette section de ligne est particulièrement exposée. Une bonne gestion de la surveillance du niveau des eaux, des prévisions analysées à temps, un arsenal de mesures prévues à l'avance doivent permettre une interruption de courte durée de l'exploitation et une remise en service difficile à prévoir mais de l'ordre de quelques jours à quelques semaines selon la gravité de la crue. 